Document downloaded from:

http://hdl.handle.net/10251/162105

This paper must be cited as:

Romero-Peña, JS.; Cardona Marcet, N. (2020). Irregular MultiFocal Reflector for Efficient mmWave Propagation in Indoor Enviroments. IEEE.

https://doi.org/10.23919/EuCAP48036.2020.9136074

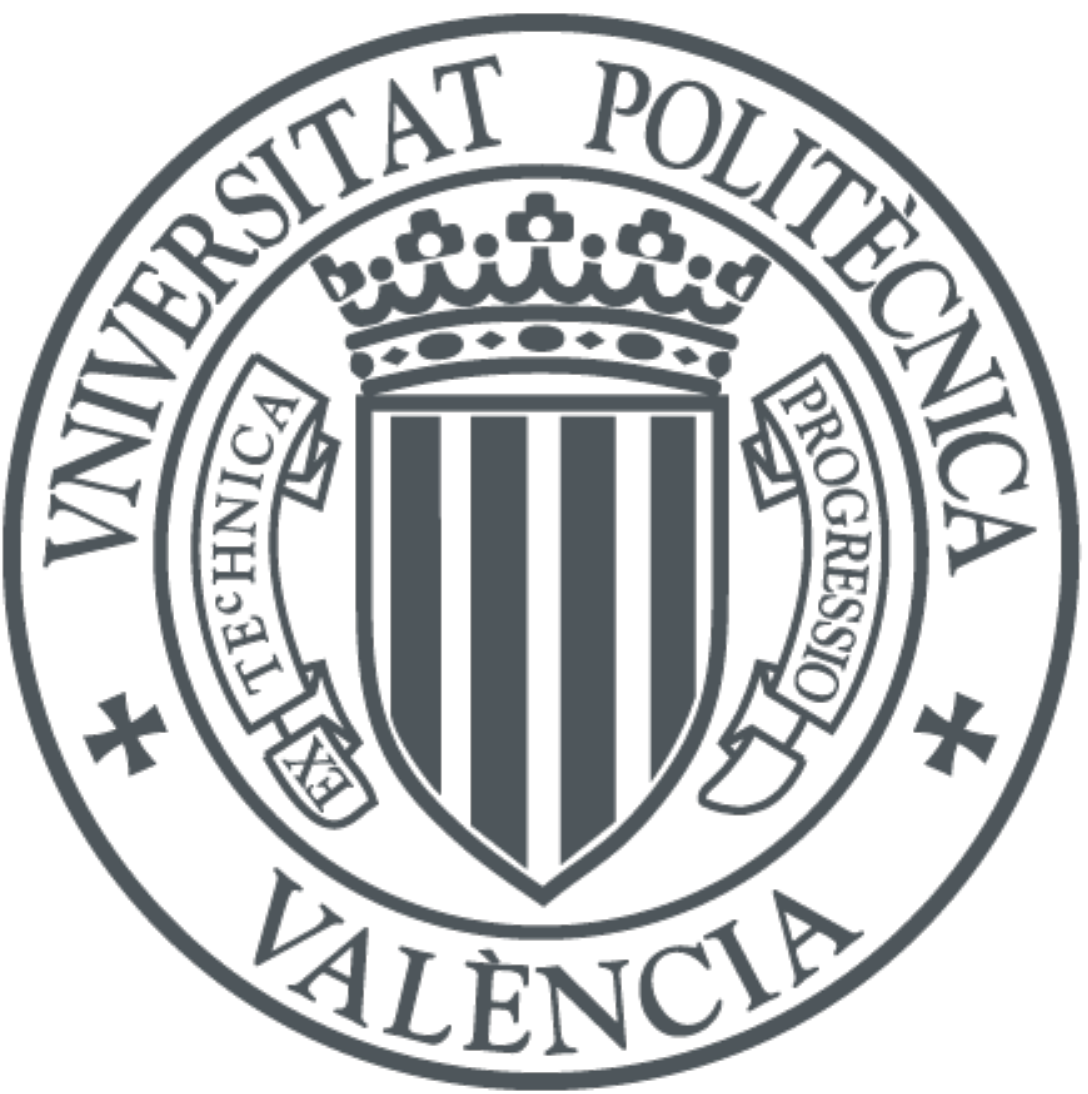

The final publication is available at

https://doi.org/10.23919/EuCAP48036.2020.9136074

Copyright IEEE

Additional Information 


\title{
Irregular MultiFocal Reflector for Efficient mmWave Propagation in Indoor Enviroments
}

\author{
J. Samuel Romero-Peña, Narcis Cardona \\ iTEAM Research Institute, Universitat Politècnica de València, València, Spain \\ $\{$ jhrope,ncardona\}@iteam.upv.es
}

\begin{abstract}
In future implementations of 5G systems, it is essential the use of the spectrum in the range of $\mathrm{mm}$-Waves frequencies, in order to offer to the users the bandwidth proposed in the standard. However, using this frequency range lead to many technical difficulties in which the most important challenge is the critical attenuation of the signal in non-line-ofsight (NLOS) environments in indoor environments. Therefore is essential to plan strategies that allow us to mitigate the problem of signal attenuation in this kind of complex environments and ensure the viability of using this technology in short term. Then the objective of this research is the design of a passive reflector that allow us to redirect the energy of the transmitting antenna efficiently in order to avoid the obstacles of the environment , and therefore avoid excessive losses .
\end{abstract}

Index Terms-5G Systems, mmWave, Reflector, Reflection , Diffuse.

\section{INTRODUCTION}

During the last years there has been a substantial increase in the demands of greater bandwidths, because today the use of the mobile phone is more widespread, not only for mobile services that consume little bandwidth such as email , calls , Twitter, What-App , etc ; as also more asymmetric use of the spectrum like the streaming uses such YouTube , Netflix , Instagram , Facebook , etc, that have more and more multimedia content [1] .

For this reason, the user does and will do a more demanding use of the spectrum and therefore the industry sees a need to have a greater bandwidth to offer a better multimedia experience. But due to technical limitations nowadays is impossible to offer this speed that is being demanded, because the spectrum is almost full. Therefore the telecommunication industry has proposed to take a technological leap and explore the spectrum in the range of mm-Waves.

One of the greatest technical challenges to offer services at the mm-Wave frequency are the very high propagation losses in free space that are an intrinsic behaviour as the frequency increases .Additionally the NLOS losses that are also high in indoor environments because all the obstacles are electrically larges [2]

Several solutions have been proposed thought in literature to avoid the issue of high signal attenuation in both indoor and outdoor environments. Some of these proposed solutions include beam-forming [2] and beam-steering techniques [3] using multiple antennas, high transmit power and high sensitivity receivers [4], and use of multiple active repeaters [5].
However, all these strategies are very complex and expensive techniques, for the reduced coverage of each base station in mm-Wave would be the equivalent of the current peak cells in the best cases $\left(100 m t^{2}\right)$ [6]. For this reason, the initial investment will be considerably high in the early stages .

In addition to the technical and coverage problems , the maximum power allowed by the telecommunications regulatory entities , for possible health problems due to energy adsorption in the human tissues at mm-Wave [7].

Therefore, and according to all these previously issues, the best technical and economic solution will be the design of a passive reflector in mm-Wave . This reflector allow to redirect the energy from the base station transmitter antenna to the user efficiently, with the aim to avoid the excessive losses and consequently increasing the coverage.

To begin with the design of this passive reflector, the starting point idea [8] was worked with a rough surface, because is the best way to increase mmWave coverage with a diffuse reflection.

To the best of the authors' knowledge there is no works in literature devoted to this issue. Although there are some studies related to reflectors for bidirectional communication links [9][13] and also studies about radar reflectors [14]-[16].

The reference paper [8] was the only one that has been considered irregular reflector surfaces for mmWaves. The paper [8] refers to a flat reflector with wedges that emulates a parabolic reflector with a fixed focal position, that can be used for satellite communications, because it reduces its volume So our goal is to adapt this idea to design a diffuse reflector according to our research.

\section{PAssive Reflector Design}

The objective of this research was to design a reflector that allow to distribute energy over a wide coverage area. Due to which the reflector cannot be a flat reflector, because in the future 5G systems at mm-Wave will be offered with very directive antennas to counteract the highest losses in the free space. Therefore with a flat reflector is the output radiation, will depend of the transmitter antenna directivity and its angle of incidence on the reflector. Therefore a flat reflector will have a specular behaviour, that is not useful for the indoor scenarios (See Figure 1 ).

So the main objective of this new passive reflector will be homogeneously spread the energy received by the transmitter 
antenna over a specific coverage region, that will be independent of the reflector location and incidence on the reflector (See Figure 1).

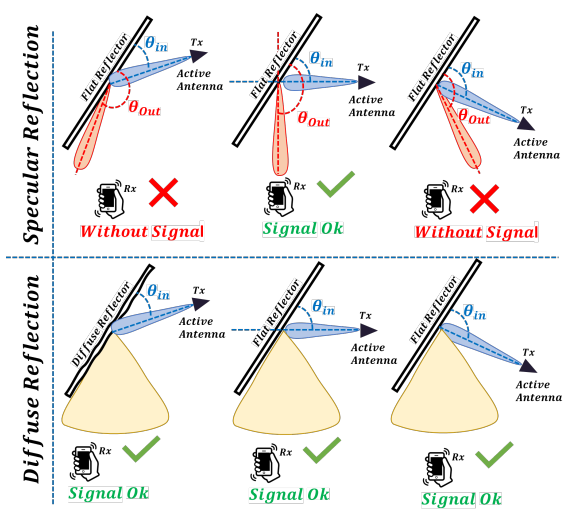

Fig. 1. Specular reflector vs Diffuse reflector for mmWave coverage management in $5 \mathrm{G}$ Systems

\section{A. Design guidelines}

Figure 2 depicts the scenario considered for this study. As can be shown, The active antenna is that highly directive array antenna that feeds the reflector. The angle of entry $\left(\theta_{i n}\right)$ is the angle at which the radiation of the active antenna hits the reflector and the coverage area $\left(\theta_{\text {coverage }}\right)$ offered by the reflector will depend on it. The output radiation $\left(\theta_{\text {out }}\right)$ refers to the angular area radiated by the reflector, which depends on the input radiation of the active antenna. The input margin $\left(\theta_{\text {in-margin }}\right)$ is the angular range in which the transmitting antenna can hit the reflector without affecting the coverage of the reflector $\left(\theta_{\text {coverage }}\right)$, according to the design guidelines.Reflector losses $\left(L_{\text {reflector }}\right)$ is the difference between the input gain $\left(G_{i n}\right)$ and the output gain $\left(G_{\text {out }}\right)$, in other words it is the maximum gain lost when dispersed by the reflector.

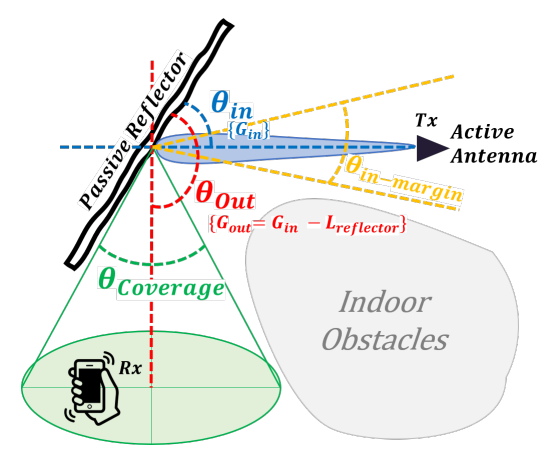

Fig. 2. Nomenclature used for reflector design guidelines

It should be taken into account that the energy should be homogeneously distributed in indoor environments. To achieve this goal, there are some guidelines that should be considered:

- The reflector must maintain a fixed coverage area of $\theta_{\text {coverage }}=40^{\circ}$, where the angle of entry can have a margin of $\left(\theta_{i n-\text { margin }}=20^{\circ}\right)$. Therefore the reflector does not have a focal position.
- The output radiation of the reflector must be homogeneous enough to assure that in a given area there is no fading of the signal of more than $6 \mathrm{~dB}$. Ensuring reflector losses $\left(L_{\text {reflector }}\right)$ not greater than $12 \mathrm{~dB}$.

- The reflector bandwidth has to be $10 \mathrm{GHz}$, in order to be versatile enough to be used in future $5 \mathrm{G}$ systems in the millimeter band. The operating range will be $30 \mathrm{GHz}$ to $40 \mathrm{GHz}$.

\section{B. Design Methodology}

Starting from the premise proposed in the paper [8], we design the reflector. One of the main requirements to start the design of the reflector was that it be a periodic, symmetrical and parametrizable surface. The surface has to be parametrizable depending on the wavelengths and the angular incidence of the radiation.

As seen in figure 3, the angle of incidence on the reflector is a critical parameter, because according to where the transmitter radiation hits the reflector, the energy will be dispersed in unwanted areas (Path B,C), losing the energy that wants to be redirected and conserve like the (Path A). It can also be observed that depending on the angle of incidence of radiation there will be areas of shadow between wedge and wedge, which reduce the effective surface and do not redirect as much energy to the area of interest (lost energy).

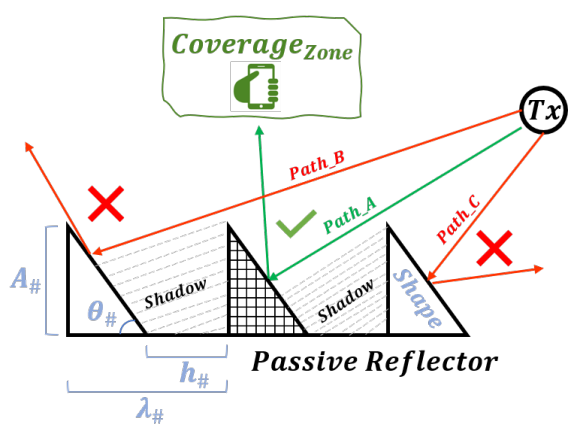

Fig. 3. Design considerations for the passive reflector

Therefore, the reflector cannot be designed lightly and it is necessary to make several iterations with different surface parameters $\left(\theta_{n}, A_{n}, h_{n}, \lambda_{n}\right.$ and Shape $\left._{n}\right)$ according to the design guidelines. The design parameters were adjusted using a ray tracing logic, trying to predict where each ray would go based on its specular reflection. Each surface was designed as a coefficient matrix of its parameters (See Figures 3,5 ), so that the reflector has a gradual behavior, in order to avoid the shadows between wedges and reflections in unwanted directions.

Therefore, different types of reflectors with different geometric shapes began to be designed, in order to identify which are with more potential .(See Figure 4). 


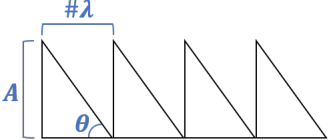

(a)

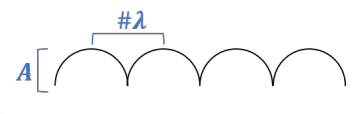

(b)

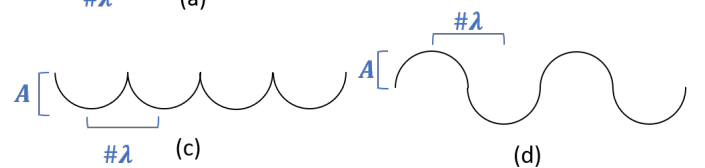

(d)

Fig. 4. Proposed geometric shapes for reflector design

\section{Simulation Methodology}

\section{A. Simulation Set-Up}

The simulations were carried out using the electronic analysis platform "CST (Computer System Technology) [20]". The methodology consisted of locating the reflector in the far field area of the transmitter, with the objective that , the incident radiation in the reflector will be a plane wave (See Figure 5). The transmitting antenna used was a horn antenna in the Ka band from $26 \mathrm{GHz}$ to $40 \mathrm{GHz}$ [19]. The reflector is an irregular metal surface that we have previously designed(See Figure 5). Once the antenna and the reflector are located, we simulate the final far field together of the two elements ( $\mathrm{Tx}+$ Reflector).

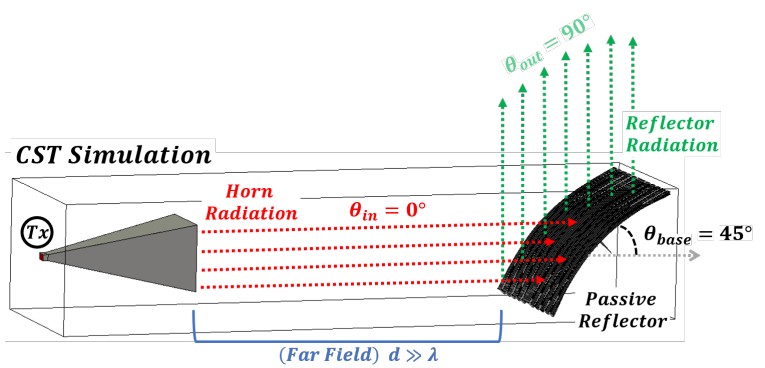

Fig. 5. Simulation scenario used to characterize reflector radiation

Therefore, the resulting far field will have the contributions of the transmitting antenna and the reflector, where the radiation pattern of the reflector will be where according to the orientation of the reflector $\theta_{\text {base }}=45^{\circ}$ with respect to the transmitting antenna $\theta_{\text {in }}=0^{\circ}$ this would have a maximum radiation at $\theta_{\text {out }}=90^{\circ}$ (See Figure 5). Therefore reflector radiation pattern results will be centered at $90^{\circ}$ (See Figure 7"Flat-Reflector").

During the testing and design process of the reflector they were tested with different types of surfaces, with different electrical periodicity of the roughnesses. This was a very arduous process because it was necessary to design several types of surfaces with a 3D professional modeling software [21]. Additionally each surface was simulated several times because we had to hit on the same surface with different angle of entry $\left(\theta_{i n}\right)$ to observe the diffuse behavior $\left(\theta_{\text {coverage }}\right)$ resulting from the reflection (See Figure 2).

\section{RESULTS}

Because part of our research work was based on paper [8], where irregular flat surfaces were designed with wedges in order to emulate parabolic reflectors. We started designing this type of reflectors in order to meet the proposed design guidelines. As previously mentioned, these surfaces were designed with a matrix of coefficients in which each coefficient determines each wedge parameter $\left(\theta_{n}, A_{n}, h_{n}, \lambda_{n}\right)$ as can be shown in figure 6-b.

Figure 6 depicts an example of one of the many flat reflectors that have been designed. where the matrix of coefficients of the slopes of each wedge is observed, and it is observed how each section of the reflector has different slope, some negative (green cells) and others positive (red cells) (See Figure Figure 6-b). Each wedge has different slopes because the objective of the reflector is to direct the energy in different positions in order to obtain a diffuse reflection.
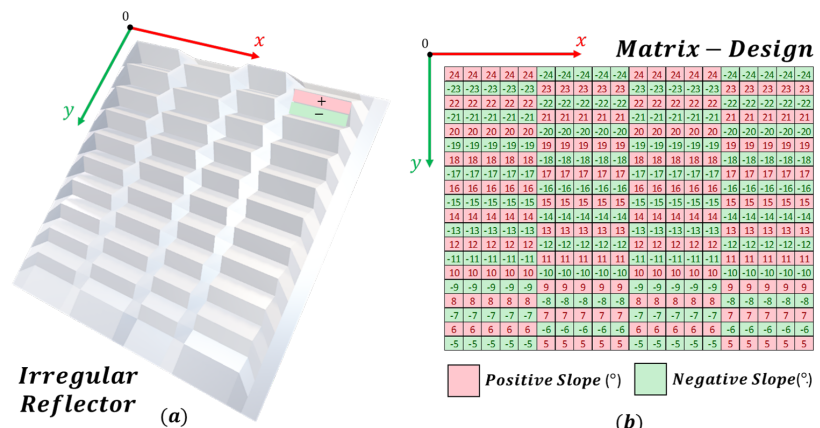

(b)

Fig. 6. Antenna design with geometric flat slopes with coefficient matrix (a)Proposed irregular reflector, (b) Coefficient matrix

In figure 7 shows the field radiated by the reflector that has been designed in figure 6 . This result is compared with the field radiated by a completely flat reflector (Red Line), where its maximum radiation follows a fully specular behavior $\theta_{\text {out }}=90^{\circ}$, and also in these results the radiation pattern of the horn antenna is observed (Blue Line) $\theta_{\text {in }}=0^{\circ}$.

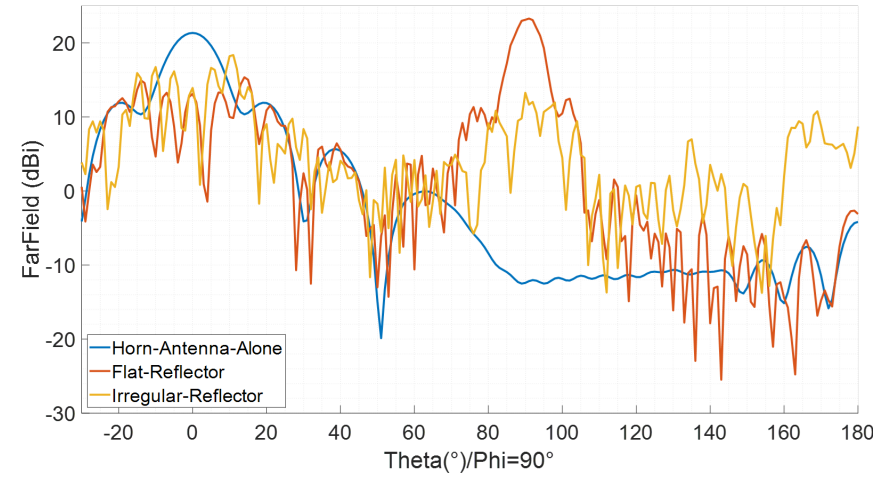

Fig. 7. Results of the radiation pattern of the irregular reflector proposed in Figure 6

After the results obtained not only from this example design, but from several designs previously considered with different 
coefficient matrices. It is concluded that for the proposed design guidelines, where a fundamental premise is that there should not be a fixed focal position. All surfaces created with flat slopes (See figure 4-a ) have a strong specular behavior, although each wedge has a different parameter to that of its adjacent. These surfaces with flat slopes had bad results, because there are usually phase problems between the contributions, which cause the resulting pattern of the reflector to attenuate the signal considerably and have strong fading as seen in the figure 7 (Yellow Line'Irregular-Reflector").

Finally after several unsatisfactory designs it was decided to design surfaces where there was a smooth transition between wedges.Surfaces with gradual and continuous slopes, in order to avoid abrupt discontinuities on the surface that could be affecting the sum consistent (in phase) of the multiple contributions of the transmitting antenna (radiation received by the reflector) . For this reason the surfaces (See figure 4b,c,d ) were proposed.

With the objective that the reflector maintains a fixed coverage area, the strategy used was to ensure that the reflector had a radiation pattern as wide as possible, in order to keep the fixed coverage area independent of the angle of incidence, while stay on the design margins $\left(\theta_{\text {in-margin }}\right)$.With this strategy, power is exchanged for coverage, between more coverage, less power and vice versa. Because the reflector is a passive element, its only function is to distribute energy efficiently, so it is important that this reflector will be supported by very high gain directive mmWave antennas.

The proposed design is in Figure 8, where it is composed of two types of surfaces. The first surface is a concave surface, where it is designed with a cosine function with a period of $0^{\circ}$ to $90^{\circ}$ with amplitude ( $\mathrm{Z}$ axis) of $15 \mathrm{~mm}$ and with dimensions of its base (X-Y axis) of $100 \mathrm{~mm} \times 100 \mathrm{~mm}$. The orientation of the second surface is orthogonal to the first surface, and is a convex surface, formed by a cosine function, where only negative cycles (convex) are considered, with a period 10 times lower than the first surface and with an amplitude ( $\mathrm{Z}$ axis) of $0.5 \mathrm{~mm}$.

This surface was not designed with a matrix of coefficients, because this surface is composed entirely of sinusoidal functions, so it does not apply to the design system proposed above for flat slope surfaces of Figure 6.

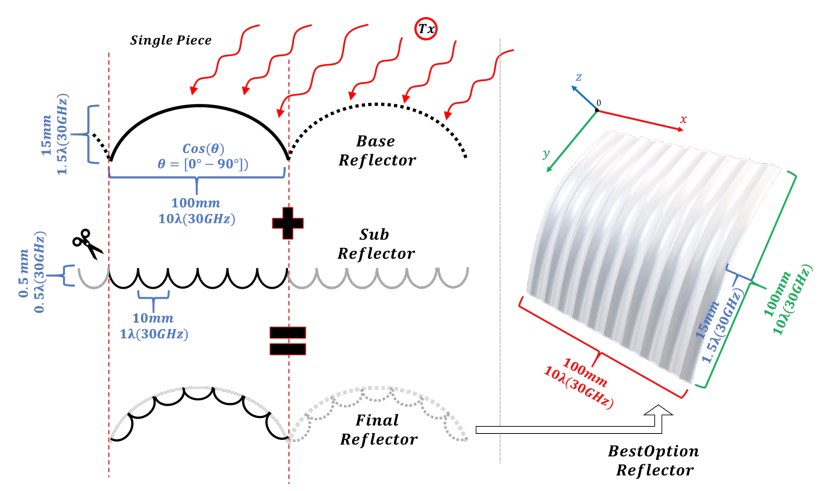

Fig. 8. Proposed design of the best reflector to improve indoor coverage
The reflector that has been designed is a periodic reflector, which means that a larger reflector may be available if the same reflector is placed consecutively. The objective of making the reflector larger will depend on the directivity of the transmitting antenna that will feed the reflector, because the less directive the antenna is, the greater the surface of the reflector should be.

This reflector design avoid that the contributions were in counter-phase and therefore that there was a considerable fading in the coverage area.The final design of the reflector is show in the Figure 9-b.

Figure 9-b shows the field radiated by the reflector that has been designed (See Figure 8). This result shows the field radiated by the reflector at $30 \mathrm{GHz}$, with different positions of the transmitting antenna(See Figure 9-a ). In each position of the $T_{x}$ this hits in the reflector different with different input angles $\theta_{\text {in }}=0^{\circ}, 10^{\circ},-10^{\circ}$, according to the design guidelines there should not be a fixed focal position, but a input range of $\theta_{\text {in-margin }}=20^{\circ}$.
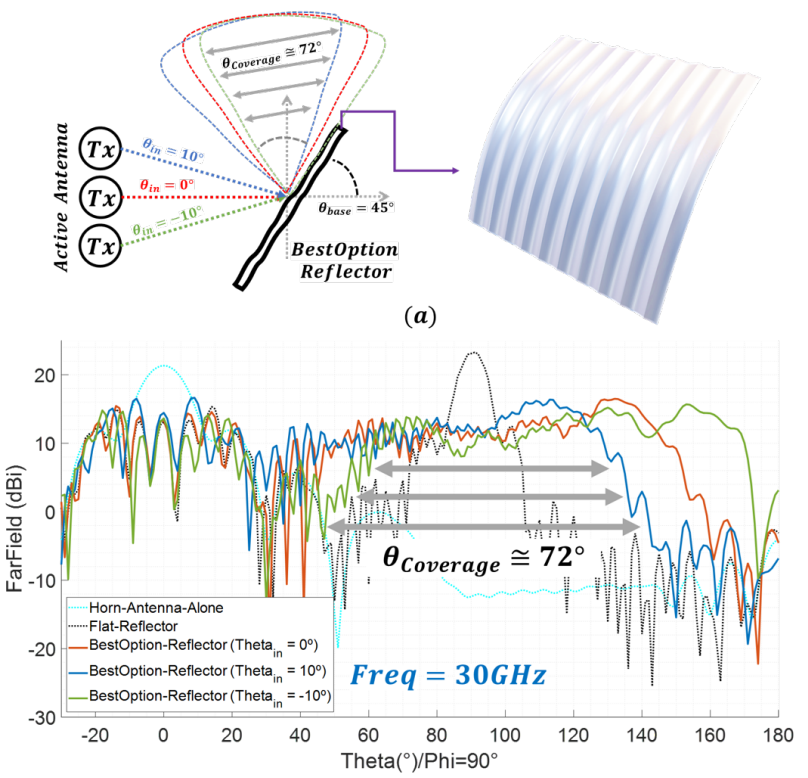

(b)

Fig. 9. (a)Simulated reflector scenario with different Tx positions.(b) 2-D radiation pattern by the diffuse reflector with different input angles $\theta_{i n}=$ $0^{\circ}, 10^{\circ},-10^{\circ}$ of $\mathrm{Tx}$ at $30 \mathrm{GHz}$

According to the results of the radiation pattern (See Figure 9-b), it is concluded that, from a specular reflection at $90^{\circ}$, with a beam width of $15^{\circ}$ to $12 \mathrm{~dB}$ and a maximum directivity of $23 \mathrm{dBi}$. The proposed diffuse reflector increased the coverage area $\left(\theta_{\text {coverage }}\right)$ from $15^{\circ}$ to $100^{\circ}$ to $12 \mathrm{~dB}$, homogeneously distributing the energy throughout the coverage range, in the case of any fixed position of the transmitter. But in the case where the angle of entry is changed in a range of $\theta_{\text {in-margin }}=20^{\circ}$, the coverage area in common becomes approximately $72^{\circ}$. In the case of this reflector, maximum gain was exchanged for coverage and flexibility. 
The following results (See Figure 10) show the behavior of the radiation pattern of the reflector, as it changes between three operating frequencies $(30 \mathrm{GHz}-35 \mathrm{GHz}-40 \mathrm{GHz})$ and different input angles $\left(\theta_{\text {in }}=0^{\circ}, 10^{\circ},-10^{\circ}\right)$ in order to determine the bandwidth proposed in the design guidelines in the mmWave spectrum.

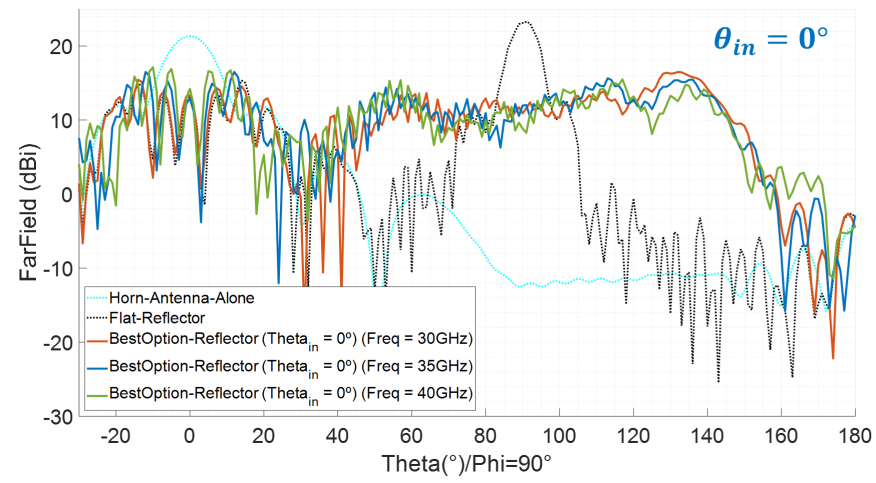

(a)

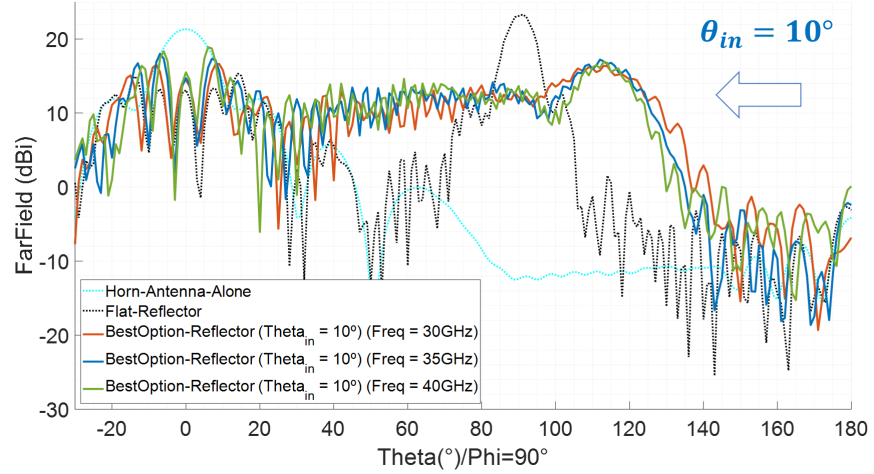

(b)

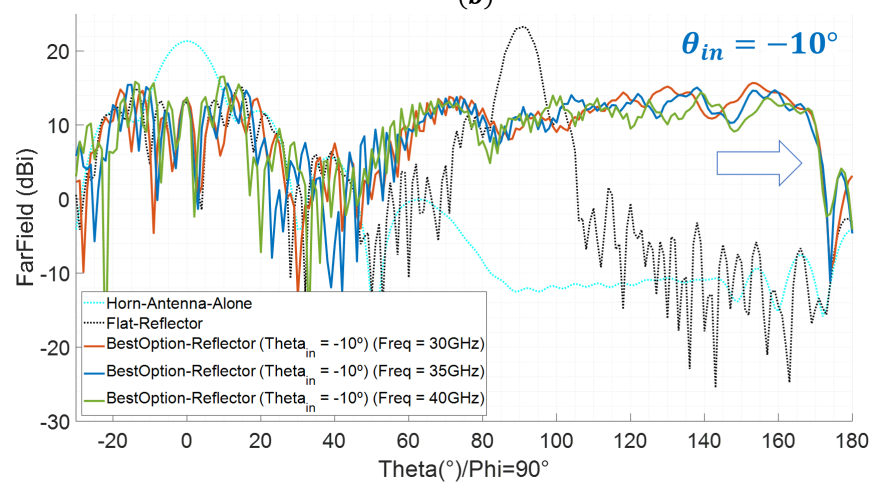

(c)

Fig. 10. Field coverage radiated by the reflector at different frequencies $(30 \mathrm{GHz}, 35 \mathrm{GHz}, 40 \mathrm{GHz})$ and input angles $\left(\theta_{i n}=0^{\circ}, 10^{\circ},-10^{\circ}\right)$

According to the previous results, it is concluded that the reflector operates in the proposed bandwidth from $30 \mathrm{GHz}$ to $40 \mathrm{GHz}$, for any position of the transmitting antenna in the range of $\theta_{\text {in-margin }}=20^{\circ}$.

\section{CONCLUSIONS}

This paper has presented a solution to distribute energy in indoor environments efficiently in the mmwave range.
Therefore, a passive reflector has been designed that distributes the received power homogeneously, increasing the coverage area by $450 \%$ in exchange for a maximum gain reduction of approximately $10 \mathrm{~dB}$ in an operating bandwidth of $10 \mathrm{GHz}$ (30GHz-40GHz ).This reflector aims to solve coverage problems in $5 \mathrm{G}$ systems in indoor environments, in order to avoid losses due to obstacles.

\section{ACKNOWLEDGMENT}

This work was supported by the H2020 Marie Curie program, with project grant no: 766231 WAVECOMBE-ITN2017

\section{REFERENCES}

[1] https://variety.com/2019/digital/news/netflix-loses-title-top-downstreambandwidth-application-1203330313/

[2] R. R. T, D. Sen and G. Das, "On Bounds of Spectral Efficiency of Optimally Beamformed NLOS Millimeter Wave Links" , in IEEE Transactions on Vehicular Technology, vol. 67, no. 4, pp. 3646-3651, April 2018.

[3] M. N. Akbar, S. Atique, M. Saquib and M. Ali, "Capacity Enhancement of Indoor 5G mmWave Communication by Beam Steering and Narrowing”, 2018 10th International Conference on Electrical and Computer Engineering (ICECE), Dhaka, Bangladesh, 2018, pp. 85-88.

[4] C. Li and C. Kuo, "16.9- $\mathrm{mW}$ 33.7-dB gain mmWave receiver front-end in 65 nm CMOS”, 2012 IEEE 12th Topical Meeting on Silicon Monolithic Integrated Circuits in RF Systems, Santa Clara, CA, 2012, pp. 179-182.

[5] D. D. Falconer, J. P. DeCruyenaere, "Coverage enhancement methods for LMDS”, IEEE Commun. Mag., vol. 41, no. 7, pp. 86-92, Jul. 2003.

[6] A. I. Sulyman, "Radio propagation path loss models for $5 G$ cellular networks in the $28 \mathrm{GHZ}$ and $38 \mathrm{GHZ}$ millimeter-wave bands", IEEE Commun. Mag., vol. 52, no. 9, pp. 78-86, Sep. 2014.

[7] https://www.fcc.gov/general/specific-absorption-rate-sar-cellulartelephones

[8] V. Manohar, J. M. Kovitz and Y. Rahmat-Samii, "Synthesis and Analysis of Low Profile, Metal-Only Stepped Parabolic Reflector Antenna", in IEEE Transactions on Antennas and Propagation, vol. 66, no. 6, pp. 27882798, June 2018.doi: 10.1109/TAP.2018.2821694

[9] Y. Huang, N. Yi, X. Zhu, ”Investigation of using passive repeaters for indoor radio coverage improvement", IEEE Ant. Propag. Society Sympos., vol. 2, pp. 1623-1626, June 2004.

[10] J. L. D. L. T. Barreiro, F. L. E. Azpiroz, ”Passive reflector for a mobile communication device”, Aug. 2006.

[11] C. C. Cutler, "Passive repeaters for satellite communication systems", Feb. 1965.

[12] J. L. Ryerson, "Passive satellite communication”,Proc. of the IRE, vol. 48, no. 4, pp. 613-619, April 1960.

[13] Y. E. Stahler, "Corner reflectors as elements passive communication satellites”, IEEE Trans. Aerospace, vol. 1, no. 2, pp. 161-172, Aug. 1963.

[14] J. Bjornholt, G. Hamman, S. Miller, ”Electronic fence using highresolution millimeter-wave radar in conjunction with multiple passive reflectors", , 2002.

[15] W. Khawaja, K. Sasaoka, I. Guvenc, "UWB radar for indoor detection and ranging of moving objects: An experimental study", Proc. IEEE Int. Workshop Ant. Technol. (iWAT), pp. 102-105, 2016.

[16] C. Bredin, J.-M. Goutoule, R. Sanchez, J.-P. Aguttes, T. Amiot, ”High resolution SAR micro-satellite based on passive reflectors", Proc. IEEE Int. Geoscience and Remote Sensing Symposium, vol. 2, pp. 1196-1199, 2004.

[17] W. Khawaja, O. Ozdemir, Y. Yapici, I. Guvenc, Y. Kakishima, "Coverage enhancement for mmWave communications using passive reflectors", Proc. IEEE Global Symp. Millimeter Waves (GSMM), May 2018.

[18] S. Hiranandani, S. Mohadikar, W. Khawaja, O. Ozdemir, I. Guvenc, D. Matolak, "Effect of passive reflectors on the coverage of IEEE 802.11ad mmWave systems", Proc. IEEE Vehic. Technol. Conf. (VTC) workshops, Aug. 2018.

[19] https://www.sagemillimeter.com/content/datasheets/SAR-2309-28S2.pdf

[20] https://www.3ds.com/products-services/simulia/products/cst-studio-suite [21] https://www.blender.org/ 